\title{
DISTRIBUIÇÃO ESPACIAL DA \\ MORBIMORTALIDADE POR CÂNCER DE PRÓSTATA EM INDIVÍDUOS ADULTOS NO ESTADO DE ALAGOAS-BRASIL
}

Spatial distribution of morbimortality by prostate cancer in adult individuals in the state of Alagoas-Brazil

Distribución espacial de la morbimortalidad por cáncer de próstata

en adultos en el estado de Alagoas-Brasil

\section{Claudio José dos Santos Júnior ${ }^{1}$, Elaine Cristina Torres Oliveira ${ }^{1 *}$}

${ }^{1}$ Núcleo de Ciências Humanas, Sociais e Políticas Públicas (NUCISP) da Universidade Estadual de Ciências da Saúde de Alagoas (UNCISAL), Maceió-AL, Brasil.

*Correspondência: NUCISP-UNCISAL. R. Dr. Jorge de Lima, 113 - Trapiche da Barra, Maceió - AL, 57010-300. E-mail: elaine.oliveira@uncisal.edu.br.

\section{RESUMO}

A Organização Mundial da Saúde reconhece o câncer como um importante problema de saúde pública. De todos os tipos de cânceres, a neoplasia maligna de próstata $(\mathrm{CaP})$ ganha considerável relevância pelo fato de ser a segunda mais frequente entre os homens no âmbito nacional, ficando atrás apenas do câncer de pele. Essa doença, apesar de estar comumente relacionada às idades mais avançadas, vem acometendo, de forma cada vez mais frequente, homens a partir de 45 anos e até mesmo antes dessa faixa etária. Este trabalho objetiva avaliar a distribuição geoespacial da incidência e mortalidade por câncer de próstata em homens adultos em um estado do Brasil. Tratase de estudo ecológico realizado a partir dos registros de morbimortalidade para câncer de próstata em homens com idade entre 20 e 59 anos no estado de Alagoas, Brasil. Foram utilizados os programas SPSS 25 e BioEstat 5.3 para estimar a variação percentual anual da incidência e da mortalidade e seus respectivos intervalos de confiança e o software Quantum GIS 3.0 para realização da distribuição espacial dos indicadores, tendo sido o padrão de distribuição espacial verificado através da elaboração de mapas de calor. Foi observado que 209 homens foram atendidos em virtude do câncer de próstata em Alagoas. Foi verificada a ocorrência 54 óbitos (25,8\%). A distribuição espacial dos casos demonstrou áreas quentes (hot spot) para de diagnósticos e óbitos em áreas urbanas. Indivíduos com diagnóstico ou óbito por câncer de próstata estiveram concentrados nas maiores cidades de Alagoas no período estudado.

Palavras-chave: Neoplasias Prostáticas. Análise Espacial. Epidemiologia.

\section{ABSTRACT}

The World Health Organization recognizes cancer as an important public health problem. Of all types of cancers, malignant prostate cancer (CaP) gains considerable relevance because it is the second most frequent among men nationwide, second only to skin cancer. This disease, despite being commonly related to older ages, has been affecting, more and more frequently, men over 45 years old and even before this age group. This work aims to evaluate the geospatial distribution of prostate cancer incidence and mortality in adult men in a Brazilian state. This is an ecological study based on morbidity and mortality records for prostate cancer in men aged between 20 and 59 years in the state of Alagoas, Brazil. The SPSS 25 and BioEstat 5.3 programs were used to estimate the annual percentage change in incidence and mortality and their respective confidence intervals and the Quantum GIS 3.0 software to perform the spatial distribution of the indicators, the spatial distribution pattern being verified through the elaboration of heat maps. It was observed that 209 men were treated for prostate cancer in Alagoas. 
The occurrence of 54 deaths (25.8\%) was verified. The spatial distribution of cases showed hot areas (hot spots) for diagnoses and deaths in urban areas. Individuals diagnosed or died from prostate cancer were concentrated in the largest cities in Alagoas during the study period.

Key-words: Prostatic Neoplasms. Spatial analysis. Epidemiology.

\section{RESUMEN}

La Organización Mundial de la Salud reconoce al cáncer como un importante problema de salud pública. De todos los tipos de cáncer, el cáncer de próstata maligno (CaP) gana una relevancia considerable porque es el segundo más frecuente entre los hombres en todo el país, solo superado por el cáncer de piel. Esta enfermedad, a pesar de estar comúnmente relacionada con edades más avanzadas, ha venido afectando, cada vez con mayor frecuencia, a hombres mayores de 45 años e incluso antes de este grupo de edad. Este trabajo tiene como objetivo evaluar la distribución geoespacial de la incidencia y la mortalidad por cáncer de próstata en hombres adultos en un estado brasileño. Se trata de un estudio ecológico basado en registros de morbilidad y mortalidad por cáncer de próstata en hombres de entre 20 y 59 años en el estado de Alagoas, Brasil. Se utilizaron los programas SPSS 25 y BioEstat 5.3 para estimar el cambio porcentual anual en incidencia y mortalidad y sus respectivos intervalos de confianza y el software Quantum GIS 3.0 para realizar la distribución espacial de los indicadores, que fue el patrón de distribución espacial verificado mediante la elaboración de mapas de calor. Se observó que 209 hombres fueron tratados por cáncer de próstata en Alagoas. Se verificó la ocurrencia de 54 muertes (25,8\%). La distribución espacial de los casos mostró áreas calientes (puntos calientes) para diagnósticos y muertes en áreas urbanas. Los individuos diagnosticados o fallecidos por cáncer de próstata se concentraron en las ciudades más grandes de Alagoas durante el período de estudio.

Palabras clave: Neoplasias prostáticas. Análisis espacial. Epidemiología.

\section{INTRODUÇÃO}

O câncer é caracterizado como um conjunto de mais de 100 doenças que têm em comum o crescimento desordenado de células com capacidade de invadir os tecidos e órgãos e de se espalhar pelas regiões do corpo. Tal agravo é considerado um relevante problema de saúde pública, especialmente entre os países de média e baixa renda como o Brasil (WORLD HEALTH ORGANIZATION, 2020).

O impacto social do câncer em termos de morbimortalidade, além de seu alto custo para os serviços de saúde, tornaram esse conjunto de doenças objeto de políticas específicas cujo foco reside no enfrentamento por meio de estratégias de promoção, prevenção, diagnóstico e tratamento, considerando as especificidades da doença nos diversos contextos socioculturais e político-econômicos (INSTITUTO NACIONAL DE CÂNCER, 2018). que ocorreram no mundo 17 milhões de casos novos de câncer sem contar os casos de câncer de pele não melanoma e 9,5 milhões de óbitos excluindo os cânceres de pele não melanoma (BRAY et al., 2018).

No Brasil, em 2020, o Instituto Nacional do Câncer, apontava uma expectativa de cerca de 620 mil casos novos para o triênio 2020-2022, 450 mil excluindo os casos de câncer de pele não melanoma (INCA, 2020). Essa realidade revela-se preocupante quando analisada sob o aspecto da evolução da doença, dos impactos econômicos com diagnóstico e terapêutica e das perdas indiretas consequentes da incapacidade produtiva do doente.

Ao analisar as estimativas de morbimortalidade para neoplasias, o câncer de próstata $(\mathrm{CaP})$ é o terceiro em incidência (após pulmão e colorretal) e o sétimo no tocante à mortalidade (após pulmão, colorretal, estômago, 
fígado, esôfago e pâncreas). No Brasil, o CaP é o de maior incidência nos homens e o segundo em mortalidade (após pulmão). As estimativas nacionais seguem a tendência mundial e o CaP é a segunda neoplasia mais frequente entre os homens no país, ficando atrás apenas do câncer de pele (WHO, 2020; INCA, 2018; BRASIL, 2016).

Apesar de o $\mathrm{CaP}$ estar comumente relacionado às idades mais avançadas, essa patologia vem acometendo, de forma cada vez mais frequente, homens a partir de 45 anos e até mesmo antes dessa faixa etária (MEDEIROS; MENEZES; NAPOLEAO, 2011; INCA, 2018; BRASIL, 2016). Ao mesmo tempo, a literatura especializada aponta que $20 \%$ dos casos dessa neoplasia são diagnosticados em estágios avançados e uma média de $25 \%$ dos pacientes evoluem para óbito (GUERRA et al., 2008; BRAGA et al., 2017).

A incidência de $\mathrm{CaP}$ no Brasil tem aumentado nos últimos anos (BRASIL, 2016). Desse modo, a identificação das características e dos fatores envolvidos no adoecimento desta população vem sendo apontada como aspecto relevante para que sejam executadas ações mais efetivas relacionadas ao diagnóstico precoce e ao enfretamento da doença, assim como para o planejamento de ações que promovam melhoria das condições de saúde e é igualmente reconhecida como medida de melhorar a qualidade de vida dos indivíduos (GUERRA et al., 2008; SOCIEDADE BRASILEIRA DE UROLOGIA, 2018).

Em pesquisa exploratória realizada através do cruzamento dos descritores "Prostate câncer" e "adult" nas bases de dados PubMed e SciELO ficou constatado que poucos estudos foram desenvolvidos no âmbito nacional com fins de investigar a ocorrência de $\mathrm{CaP}$ e a mortalidade por essa patologia em indivíduos do sexo masculino na faixa etária dos adultos jovens. Assim, neste trabalho, visando contribuir para o conhecimento da situação de saúde deste segmento populacional, analisamos o perfil geoespacial dos indivíduos com diagnóstico ou óbito por $\mathrm{CaP}$ na população de homens adultos (20 a 59 anos) em uma unidade federativa do Brasil, o estado de Alagoas.

Essa pesquisa faz parte de um estudo mais amplo que teve como finalidades avaliar características sociodemográficas, geoespaciais e a tendência temporal dos indicadores epidemiológicos do CaP na população da Unidade Federativa (UF) em estudo.

\section{MÉTODOS}

\section{Delineamento do estudo}

Trata-se de um estudo ecológico de séries temporais, com caráter retrospectivo, que considera como unidade de análise o estado de Alagoas, Brasil. No Quadro 1 apresentamos uma síntese do desenho metodológico deste estudo.

\section{Área do estudo}

Esse estudo tem como área de abrangência o estado de Alagoas, Nordeste, Brasil. O estado faz limites com Pernambuco, Sergipe, Bahia e é banhado pelo oceano Atlântico. A extensão territorial da UFé de 27.767,661 quilômetros quadrados, sendo o território é composto por 102 municípios. A UF possuí população estimada 3.337.357 e densidade demográfica de 112,33 $\mathrm{hab} / \mathrm{km}^{2} \quad$ (INSTITUTO BRASILEIRO DE GEOGRAFIA E ESTATISTICA, 2019). 
Quadro 1. Desenho metodológico do estudo.

Objetivo: Analisar o perfil geoespacial dos indivíduos com diagnóstico ou óbito por $\mathrm{CaP}$ na população de homens adultos (20 a 59 anos) no estado de Alagoas, Brasil.

Agravo de interesse: Câncer de Próstata $(\mathrm{CaP})$.

População: Homens adultos (20 a 59 anos).

Abrangência: Estado de Alagoas (Nordeste, Brasil).

Período: 2010 a 2015.

Critérios de Inclusão: Todos os casos de diagnósticos e de óbitos por $\mathrm{CaP}$ em homens de 20 a 59 anos residentes em uma das 102 cidades do estado de Alagoas.

Técnicas: Análise espacial e análise de tendência dos indicadores.

Figura 1. Regiões de Saúde do Estado de Alagoas.

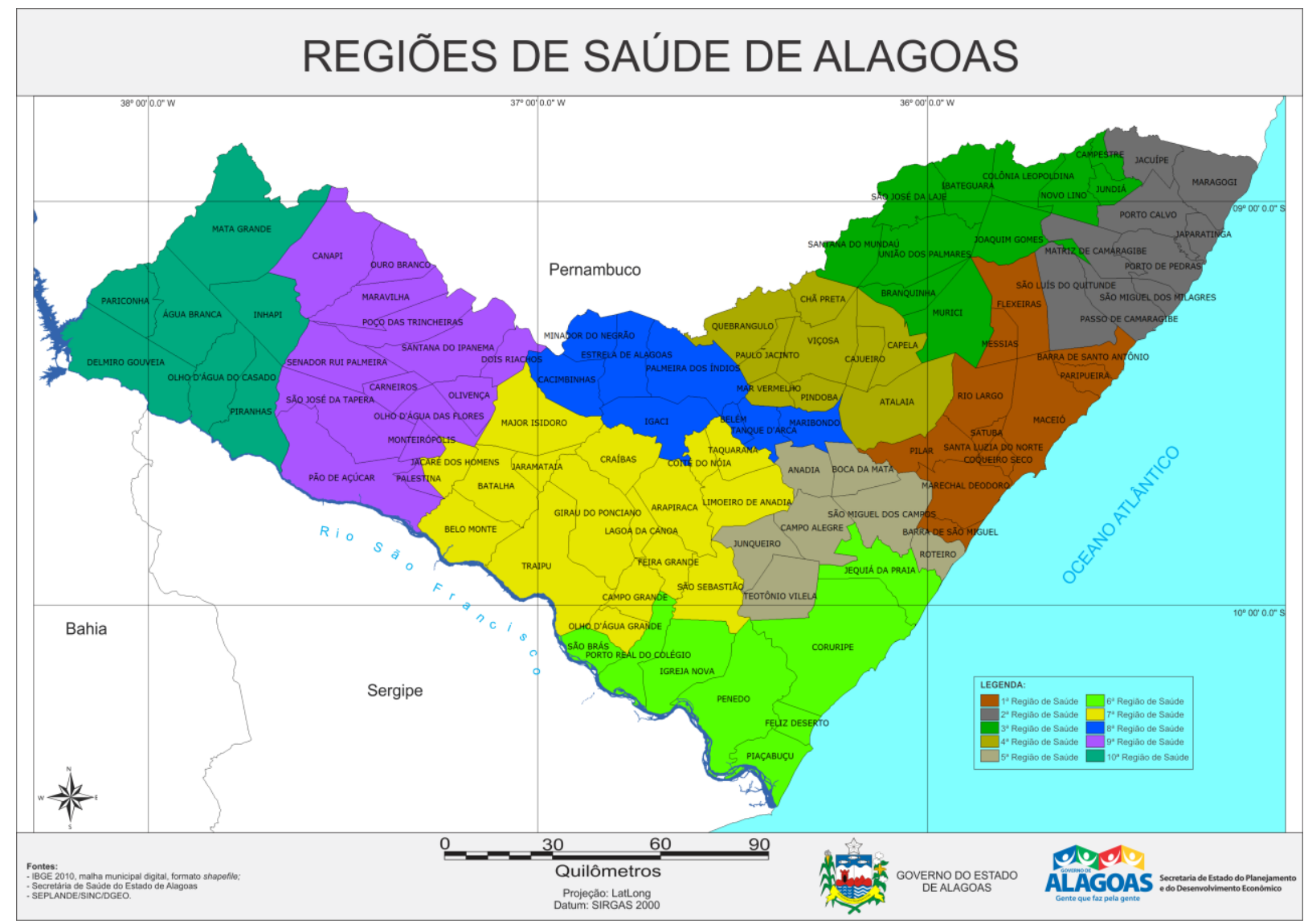

Fonte: Secretaria de Estado do Planejamento, Gestão e Patrimônio de Alagoas (2019).

\section{População e fonte de dados}

A população do estudo foi composta por todos os registros de morbimortalidade (diagnósticos e óbitos) por neoplasia urológica do tipo câncer de próstata (CID 10: C61). Foram incluídos todos casos novos no período de $1^{\circ}$ de 
janeiro de 2010 a 31 de dezembro de 2015, em homens adultos, com idade entre 20 e 59 anos.

Para consolidação das informações sobre morbidade utilizamos o IntegradorRHC, módulo do sistema de informatização dos dados sobre Registro Hospitalar de Câncer do Instituto Nacional de Câncer (SisRHC). Essa ferramenta é um sistema Web desenvolvido pelo INCA para consolidação de dados provenientes dos Registros Hospitalares de Câncer de todo o Brasil, sendo a fonte oficial de informações do Ministério da Saúde neste segmento. O preenchimento do SisRHC é obrigatório para todos os hospitais habilitados na Atenção Especializada em Oncologia do Sistema Único de Saúde (SUS) ou para aquelas unidades credenciados pelo SUS para tratamento de pacientes com financiamento público. Em Alagoas fazem parte dessa rede as seguintes unidades de saúde: Hospital Afra Barbosa; Centro Hospitalar Manoel André; Hospital Universitário Prof. Alberto Antunes; Santa Casa de Misericórdia de Maceió. Os dados sobre mortalidade, por sua vez, foram obtidos por meio do Sistema de Informações sobre Mortalidade (SIM) por intermédio da plataforma TABWIN do Ministério da Saúde.

\section{Coleta e processamento de dados}

A coleta de dados foi realizada entre os meses novembro de 2017 e janeiro de 2018. Optouse pelo ano de 2015 como ponto de corte desta pesquisa pelo fato de, quando da etapa definida em cronograma para a realização da coleta de dados, esse ter sido o último ano em que as notificações do RHC/INCA haviam sido totalmente processadas.

Os dados foram compilados no Microsoft Office Excel 2010, sendo posteriormente transportados para o programa Statistical Package for the Social Sciences 25.0 (SPSS) e BioEstat 5.3, onde foram analisados por meio da geração de indicadores descritivos e inferenciais das variáveis.

$\mathrm{Na}$ análise de tendência temporal, foram utilizados os procedimentos de regressão linear generalizada de Prais-Winsten, que considera a autocorrelação serial. Para isso, estimou-se a variação percentual anual (em inglês, anual percent change [APC]) e seus respectivos intervalos de confiança (IC95\%). Os valores de $\mathrm{p}$ foram obtidos por meio do teste de t. Foi adotado um nível de significância de $5 \%$.

A distribuição espacial das informações foi construída por meio do programa Quantum GIS 3.0 (QGIS) e levou em consideração os casos de CaP diagnosticados e a taxa de mortalidade pelo agravo nos municípios do estado. Foram considerados para representação das informações espaciais o limite dos 102 municípios do Estado. A base cartográfica do estado foi obtida junto ao Instituto Brasileiro de Geografia e Estatística (IBGE) e levou em consideração o sistema Mercator Transversal Universal e o DATUM SIRGAS 2000.

Para representação dos dados geográficos foram construídos cartogramas no modelo espacial de representação poligonal (mapas de cores) e diagramas de Kernel. Os indicadores de morbidade e mortalidade no formato de tabelas eletrônicas foram importados para camadas vetoriais por meio do comando união de tabelas na aba propriedades do arquivo no software QGIS, seguidos pela organização da simbologia (mapas de cores). $\mathrm{Na}$ construção dos diagramas de Kernel foram ponderadas as densidades em cada município com algum caso ou óbito por $\mathrm{CaP}$, aplicando o estimador de densidade de Kernel.

Tal método consiste em um interpolador empregado para estimar a intensidade do evento em uma região e tem como característica a execução de 
uma estatística de estimação de curvas de densidade, exposto por desenhos circulares nas imediações de cada ponto amostral (SOUZA et al., 2013). Como processo inicial para confecção deste mapa, foi criada uma malha de pontos, com a disposição de um ponto por município, sendo este, representado por um valor atribuído ao número de casos notificados pelo SisRHC/INCA ou pelo SIM.

Após a criação desta malha, aplicou-se o estimador de Kernel (função: uniforme), por meio da opção caixa de ferramentas de processamento, na função interpolar e mapa de calor, nesta devida ordem. Seguidamente, definindo a camada de pontos, delimitou-se o raio espacial de $50 \mathrm{~km}$, estimando a distância em relação ao valor atribuído ao ponto, originando um arquivo em formato raster. Em seguida, editou-se o número de classes e o gradiente de cores, e converteu-se o arquivo para a modalidade vetorial.

\section{Aspectos éticos}

Por se tratar de estudo realizado com o uso de dados oriundos de fontes secundárias, sem possibilidade de identificação dos pacientes e/ou de seus dados pessoais, não foi necessária a aprovação pelo Comitê de Ética em Pesquisa, conforme Resolução no 466/2012 do Conselho Nacional de Saúde.

\section{RESULTADOS}

Foi observado entre os atendimentos hospitalares ocorridos no estado de Alagoas, no período de 2010 a 2015, que 317 homens, com idade entre 20 e 59 anos, obtiveram diagnóstico de neoplasia maligna. Destes, 209 (65,9\%) foram diagnosticados como casos novos de CaP. Diante dos registros no SisRHC/INCA, foi observada uma taxa de incidência específica para $\mathrm{CaP}$, entre homens de 20 a 59 anos, no período de 2010 a 2015 de aproximadamente 25 casos para cada 100.000 homens e média de 4,2 casos ao ano.

A Tabela 1 apresenta a evolução temporal das taxas de incidência de $\mathrm{CaP}$ no estado de Alagoas. Observa-se um crescimento de 8,5\% na taxa de incidência de casos de CaP nos homens de 20 a 59 anos, entre 2010 e 2015 , tendo em vista que passou de 4,7/100 mil habitantes em 2010 para 5,1/100 mil habitantes em 2015, com tendência crescente e significativa.

Tabela 1. Tendência da taxa de incidência de casos de câncer de próstata, em homens de 20 a 59 anos, no período de 2010 a 2015, Alagoas, Brasil. (N=209).

\begin{tabular}{lccccccccc}
\hline \multicolumn{10}{c}{ Série temporal } \\
\hline $\mathbf{2 0 1 0}$ & $\mathbf{2 0 1 1}$ & $\mathbf{2 0 1 2}$ & $\mathbf{2 0 1 3}$ & $\mathbf{2 0 1 4}$ & $\mathbf{2 0 1 5}$ & APCa (\%) & IC95\% & p-valor & Tendência \\
4,7 & 2,9 & 2,6 & 5,3 & 4,2 & 5,1 & $8,5 \%$ & $2,937-5,329$ & $<0,001$ & Aumento \\
\hline APCa: annual percent change ou variação percentual anual. & \\
IC: Intervalo de confiança. \\
Fonte: Registro Hospitalar de Câncer do Instituto Nacional do Câncer (RHC/INCA).
\end{tabular}

A análise da distribuição espacial dos casos de $\mathrm{CaP}$ no período estudado verificou grande dispersão estadual dos indivíduos acometidos pela doença, concentrando-se o maior número de casos nos municípios de Maceió (n=96) e Arapiraca $(n=32)$. Na Figura 2, é possível observar a análise 
da distribuição dos casos novos de $\mathrm{CaP}$, no estado de Alagoas, no período de 2010 a 2015, segundo local de residência dos indivíduos diagnosticados. Verifica-se que as maiores proporções de casos novos no estado de Alagoas, no período de 2010 a 2015, foram de indivíduos residentes no município de Maceió (55,3\%, 50,0\%, 40,9\%, 42,2\%, 41,7\%, $36,4 \%$, respectivamente).

Figura 2. Distribuição espacial da proporção de casos de câncer de próstata, segundo local de residência, no estado de Alagoas, Brasil, 2010-2015 (N=209).
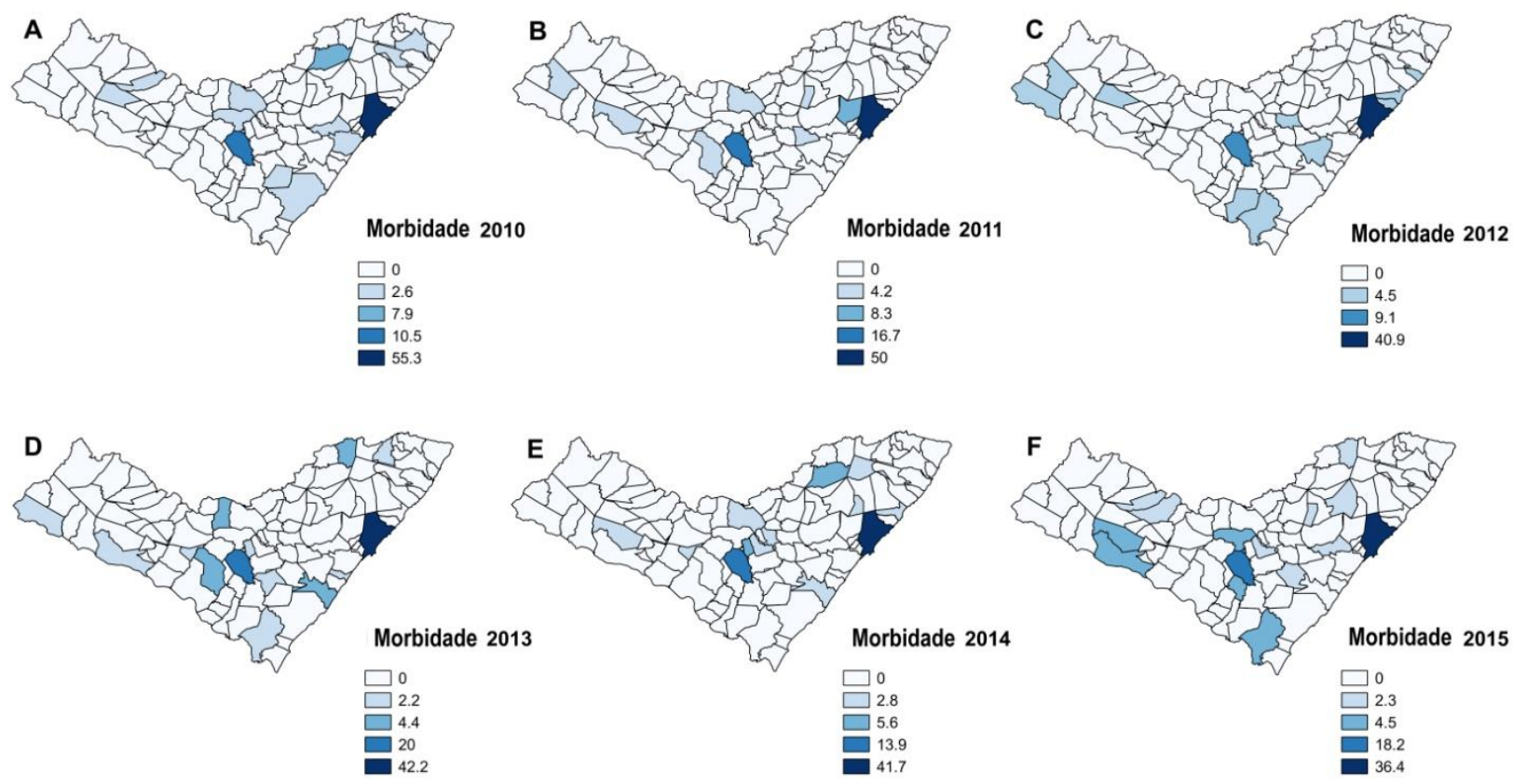

Fonte: Dados da Pesquisa, 2018.

Ao utilizar o estimador de densidade de Kernel (Figura 3) verificou-se uma maior intensidade de incidência de $\mathrm{CaP}$ (hot spot) para dois municípios do estado, Maceió e Arapiraca, localizados na $1^{\text {a }}$ e na $7^{\text {a }}$ Regiões de Saúde, respectivamente.

Diante dos registros no SIM/DATASUS, foi observada uma taxa de mortalidade específica para $\mathrm{CaP}$, entre homens de 20 a 59 anos, no período de 2010 a 2015 de aproximadamente 6,5 para cada
100.000 homens representada por 54 óbitos. A tabela 2 apresenta a evolução temporal das taxas de mortalidade de $\mathrm{CaP}$ no estado de Alagoas no segmento estudado. Em análise, observa-se um crescimento de $77,8 \%$ na taxa de mortalidade por $\mathrm{CaP}$ nos homens no período investigado, tendo a taxa de mortalidade para câncer de próstata passado de 0,9/100 mil habitantes em 2010 para 1,6/100 mil habitantes em 2015, com tendência crescente e significativa. 
Figura 3. Mapa do estimador de Kernel sobre a densidade dos casos de câncer de próstata no estado de Alagoas, Brasil, 2010-2015.

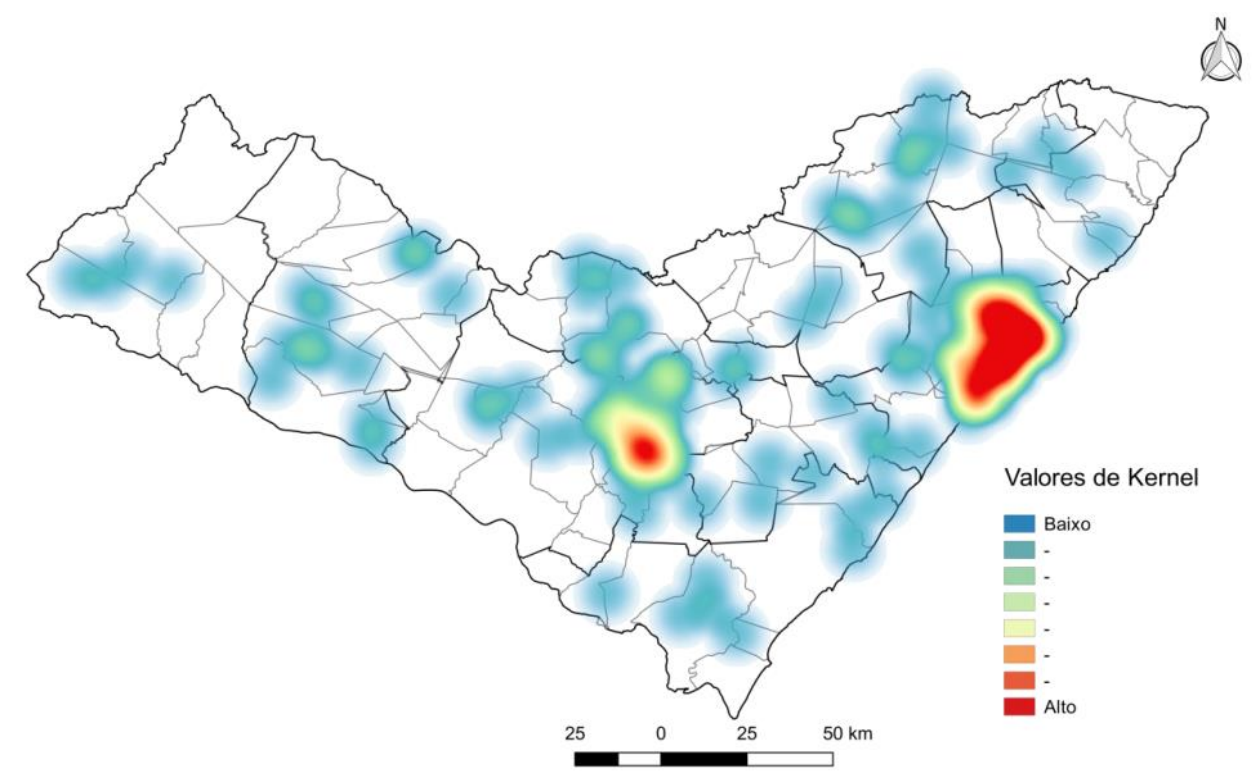

Fonte: Dados da Pesquisa, 2018.

Tabela 2. Tendência da taxa de mortalidade para câncer de próstata, em homens de 20 a 59 anos, no período de 2010 a 2015, Alagoas, Brasil. (N=54).

\begin{tabular}{cccccccccc}
\hline \multicolumn{10}{c}{ Série temporal } \\
\hline $\mathbf{2 0 1 0}$ & $\mathbf{2 0 1 1}$ & $\mathbf{2 0 1 2}$ & $\mathbf{2 0 1 3}$ & $\mathbf{2 0 1 4}$ & $\mathbf{2 0 1 5}$ & APCa (\%) & IC95\% & p-valor & Tendência \\
0,9 & 1,1 & 1,3 & 0,6 & 0,9 & 1,6 & $77,8 \%$ & $0,699-1,434$ & 0,001 & Aumento \\
\hline
\end{tabular}

APCa: annual percent change ou variação percentual anual.

IC: Intervalo de confiança.

Fonte: Sistema de Informação sobre Mortalidade do Sistema Único de Saúde (SIM/DATASUS).

A análise da distribuição espacial da mortalidade por $\mathrm{CaP}$ em adultos revelou que dos 102 municípios do estado de Alagoas, 23,5\% $(n=24)$ apresentaram registros de óbitos por este agravo na população de homens de 20 a 59 anos. A Figura 4 demonstra a distribuição espacial de todos os óbitos notificados por $\mathrm{CaP}$ no segmento em estudo por município de residência no decorrer dos seis anos. Foi observado que Maceió concentrou as maiores frequências de mortalidade durante o período estudado $(71,4 \% ; 33,3 \% ; 45,5 \% ; 20 \%$; $25 \% ; 28,6 \%$, respectivamente). 
Figura 4. Distribuição espacial da mortalidade por câncer de próstata, segundo local de residência, no estado de Alagoas, Brasil, 2010-2015 (N=54).
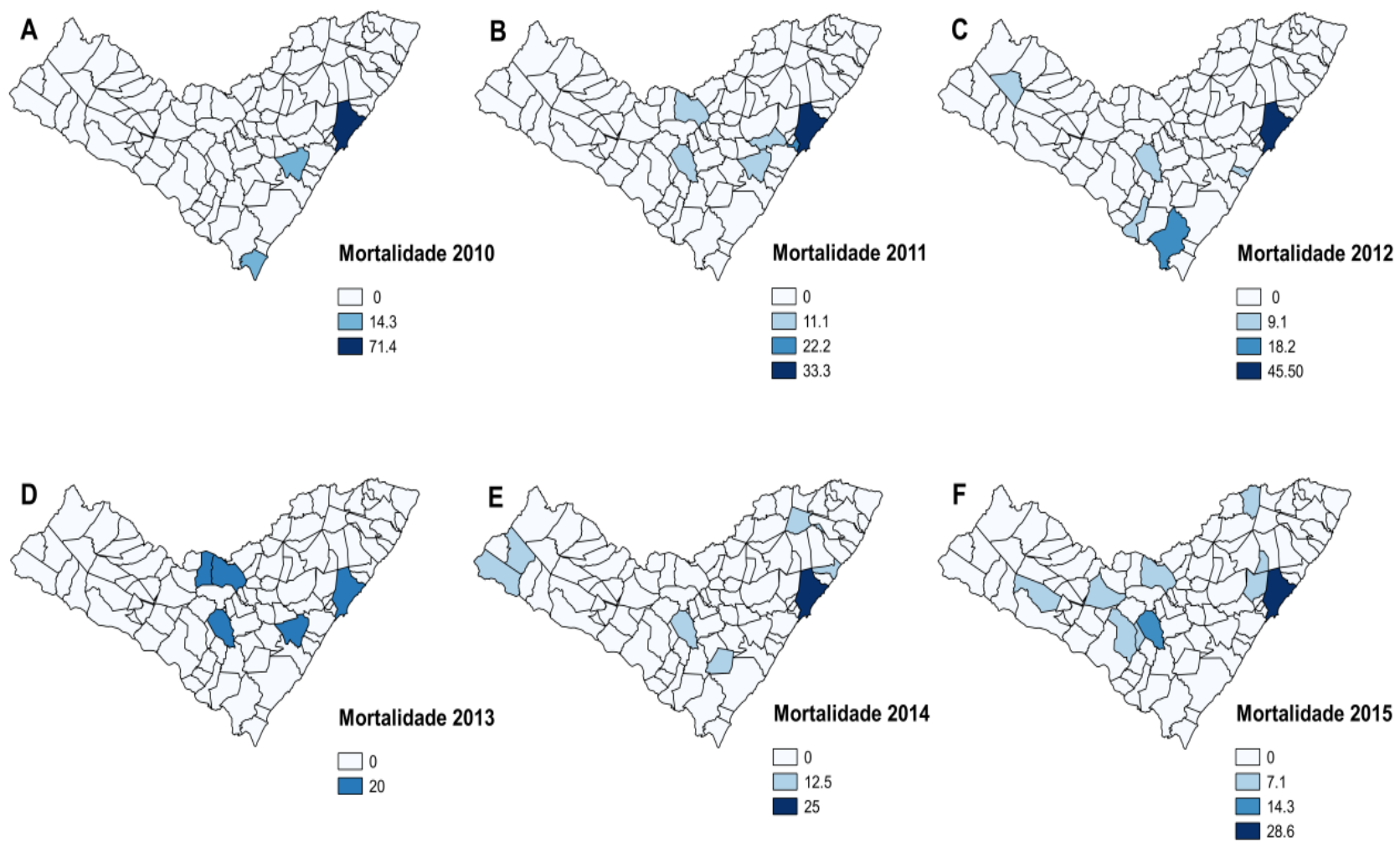

Fonte: Dados da Pesquisa, 2018.

Ao agrupar os municípios por regiões de saúde, observou-se que a $1^{\mathrm{a}}$ mesorregião sanitária foi aquela que apresentou maior número de cidades com registro de óbito por $\mathrm{CaP}$, totalizando seis municípios (Barra de Santo Antônio, Barra de São Miguel, Maceió, Messias, Pilar, Rio Largo). Além desses, outros dezoito municípios apresentaram registro de óbito por $\mathrm{CaP}$ em indivíduos adultos: Ibateguara, Joaquim Gomes ( $3^{\mathrm{a}}$ mesorregião); São Miguel dos Campos, Teotônio Vilela (5 mesorregião); Coruripe, Penedo, Piaçabuçu, Porto Real do Colégio (6 $6^{\mathrm{a}}$ mesorregião); Arapiraca, Girau do Ponciano, Lagoa da Canoa, Major Isidoro ( $7^{\mathrm{a}}$ mesorregião); Estrela de Alagoas, Palmeira dos
Índios ( $8^{\mathrm{a}}$ mesorregião); São José da Tapera ( $9^{\mathrm{a}}$ mesorregião); Água Branca, Delmiro Gouveia, Inhapi (10 mesorregião). Não foi registrada a ocorrência de óbito por $\mathrm{CaP}$ no público alvo deste trabalho, nos anos em análise, em nenhuma das cidades da $2^{\mathrm{a}}$ e $4^{\mathrm{a}}$ mesorregiões sanitárias do estado de Alagoas.

Ao utilizar o estimador de densidade de Kernel (Figura 5) verificou-se maior intensidade de mortalidade por $\mathrm{CaP}$ (hot spot) para uma única área entre as regiões de saúde do estado, na $1^{\text {a }}$ mesorregião de saúde. 
Figura 5. Mapa do estimador de Kernel sobre a densidade dos óbitos por câncer de próstata no estado de Alagoas, Brasil, 2010-2015.

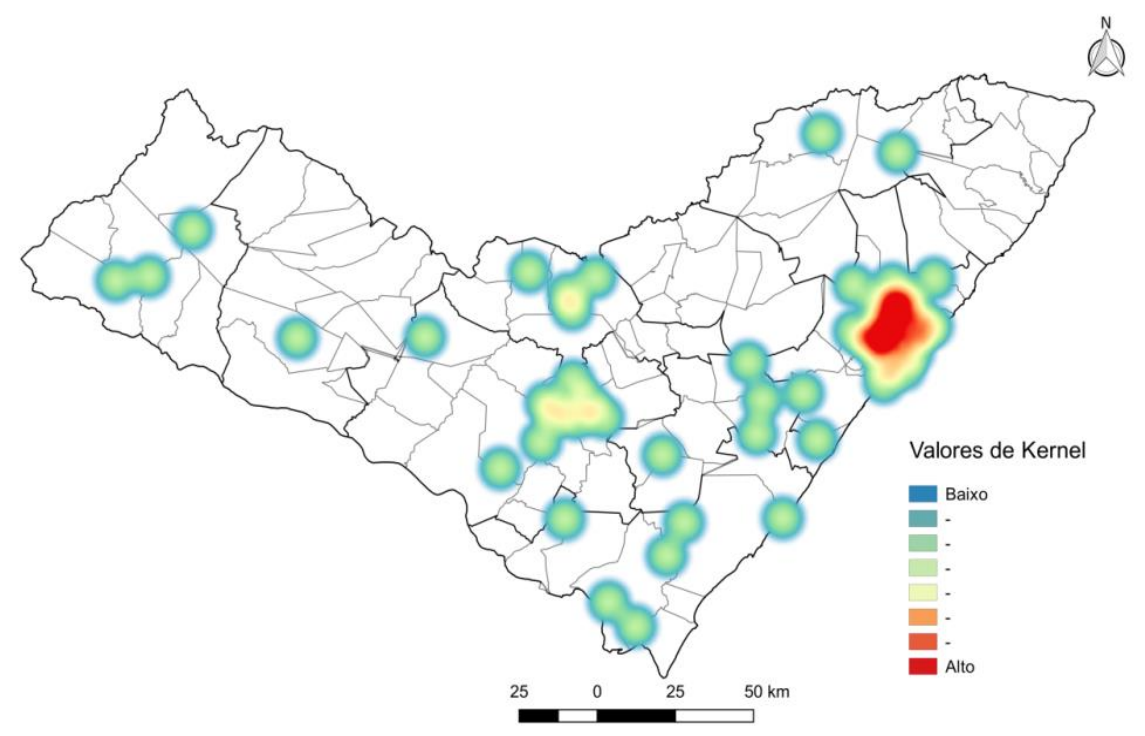

Fonte: Dados da Pesquisa, 2018.

\section{DISCUSSÃO}

$\mathrm{O}$ CaP é uma das neoplasias malignas que mais acometem homens em todas as regiões do Brasil (INCA, 2018). Em virtude da tendência de crescimento dos casos e óbitos e da representatividade dessa patologia nas condições de saúde da população masculina, essa doença tem impulsionado ações e políticas públicas, com foco de prevenção, correção e de promoção da saúde desses indivíduos (BRASIL, 2009).

Considerando que a maior concentração dos casos de $\mathrm{CaP}$ em homens de idade mais avançadas, grande parte dos estudos sobre o $\mathrm{CaP}$ concentram seus esforços nas estimativas e nos aspectos envolvidos para este segmento populacional (AMORIM et al., 2011; VIANA et al., 2014; CZORNY et al., 2017; BRAGA et al., 2017; LIMA et al., 2018; SOUZA et al., 2019). Poucos são os estudos que buscam identificar a tendência do $\mathrm{CaP}$ em homens mais jovens, sendo a justificava a baixa incidência nesse segmento. No entanto, é preciso evidenciar que o $\mathrm{CaP}$ apresenta longo seguimento e, com isso, torna-se necessário um olhar atento também para a saúde do homem de faixa etária mais jovem.

Nesta perspectiva, este estudo buscou identificar a morbimortalidade por $\mathrm{CaP}$, em homens de 20 a 59 anos, no estado de Alagoas. Foi observado que no período estudado, a proporção de casos novos de $\mathrm{CaP}$ nesta faixa etária, em Alagoas, foi a menor quando comparada aos demais estados do Nordeste, considerando em números absolutos (Bahia=2.757; Pernambuco=850; Ceará=789; Rio Grande do Norte $=651$; $\quad$ Maranhão $=387$; Paraíba=344; Piauí=285; Sergipe=216) (INCA, 2015). Além das informações sobre a morbidade, a identificação das características de mortalidade das populações é essencial para a organização dos serviços de saúde. Quanto aos óbitos por $\mathrm{CaP}$, identificamos que a proporção da mortalidade por $\mathrm{CaP}$ foi a segunda menor quando comparada aos demais estados do Nordeste, considerando em 
números absolutos (Bahia=323; Pernambuco=200; Ceará=150; Maranhão=97; Paraíba=87; Piauí=62; Rio Grande do Norte=59; Alagoas=54; Sergipe=40) (BRASIL, 2016). Entre as características epidemiológicas envolvidas com os diagnósticos e óbitos por $\mathrm{CaP}$, este estudo verificou que as maiores proporções estiveram relacionadas aos homens residentes na $1^{\mathrm{a}}$ e $7^{\mathrm{a}}$ regiões de saúde de Alagoas.

Levando em consideração que o $\mathrm{CaP}$ tem curso prolongado e que a presença de sinais e sintomas pode ocorrer a partir do comprometimento do órgão afetado (DAMIÃO et al., 2015) o menor número de casos de $\mathrm{CaP}$ na população estudada pode estar relacionado a uma subnotificação dos casos. Desta feita, é importante que os profissionais de saúde estejam atentos durante os cuidados à saúde do homem, para o diagnóstico precoce da doença (CZORNY et al., 2017).

A análise de tendência temporal da morbimortalidade para $\mathrm{CaP}$ entre homens de 20 a 59 anos no estado de Alagoas no período de 2010 a 2015 revelou que, apesar de apresentar pequena incidência e mortalidade, os números de casos novos e óbitos sofreram variações anuais crescentes (SANTOS JÚNIOR; OLIVEIRA, 2020). Esse achado traduz a necessidade de vigilância da doença entre homens neste segmento populacional, principalmente naqueles indivíduos com quadro clínico e fatores epidemiológicos que evidenciem maior risco de acometimento pela patologia. A Sociedade Brasileira de Urologia recomenda individualizar a abordagem e direcionar a identificação de pacientes com risco de desenvolver a doença de forma mais agressiva, considerando, por exemplo, fatores como idade, raça e história familiar (SBU, 2018). Dois estudos que avaliaram tardiamente os resultados quanto à mortalidade câncer-específica mostraram vantagem a favor desses programas e revelaram diminuição da taxa de mortalidade de $25 \%$ a $31 \%$ e de $27 \%$ a $32 \%$ quando da intervenção de forma precoce em comparação com nenhum rastreamento (TSODIKOV et al., 2017).

A distribuição espacial das informações permite a identificação visual da localização da ocorrência dos casos, viabilizando a identificação de áreas de maior e menor ocorrência, agregados espaciais e análise de heterogeneidade territorial de agravos (SANTOS; SOUZA, 2007). Houve maior concentração de diagnósticos da doença em Maceió, capital do estado, seguida de valores também relevantes para a quantidade de diagnósticos no município de Arapiraca, segunda maior cidade em habitantes, informações que foram corroboradas por meio da identificação de áreas quentes na análise de densidades de Kernel.

Uma possível relação desta distribuição espacial dos casos de CaP no estado de Alagoas se deve ao maior número de habitantes tanto em Maceió como em Arapiraca, quando comparados aos outros municípios do estado (IBGE, 2019) e ao o maior acesso ao diagnóstico e ao tratamento da doença nos centros de maior número populacional. No entanto, importante destacar que Maceió, apesar de concentrar as maiores incidências da doença no estado, apresentou declínio de tais índices nos anos analisados, com resultante redistribuição da frequência de casos para outros municípios do estado.

O estudo apresenta limitações próprias das pesquisas que utilizam dados secundários, a saber: o uso de dados secundários não permite ao pesquisador controle de possíveis erros decorrentes de digitação, registro, omissão no preenchimento dos campos e possíveis subnotificações. É possível, por exemplo, que frente às dificuldades para o diagnóstico e tratamento em sua região de referência/moradia, alguns pacientes possam ter se 
mudado temporariamente para outros municípios que têm melhor infraestrutura médica e, com isso, declarado endereço de residência divergente do seu local de moradia primário. Essa é uma das limitações do tipo de estudo empregado e que pode ter influenciado em algum grau de confundimento ou viés ao trabalho.

Porém, mesmo com essas considerações, é importante salientar que: I) a base de dados empregada se trata de instrumento oficial nacional, sendo seu preenchimento obrigatório em todos os serviços de saúde que recebem recursos do SUS para tratamento de pacientes oncológicos; II) as fontes de dados aqui empregadas são as mesmas que são utilizadas pelos órgãos de saúde para estabelecimento de projeções de incidência de câncer e consequetente direcionamento de ações de saúde; III) os estudos ecológicos são uma alternativa de baixo custo e rápida execução para estabelecer correlações de interesse para a saúde pública. Assim, as informações aqui apresentadas são relevantes e poderão auxiliar na condução, planejamento, tomada de decisão e aperfeiçoamento de ações de saúde voltadas à vigilância contínua e sistemática do agravo estudado nesta população.

\section{CONCLUSÃO}

Este estudo observou que o perfil de morbimortalidade relacionado ao câncer de próstata em homens de 20 a 59 anos, no período de 2010 a 2015, apresentou maiores proporções de acometimento em indivíduos que, espacialmente, estavam concentrados nas maiores cidades do estado de Alagoas. A análise permitiu identificar diferenças regionais que, possivelmente, são influenciadas por práticas diagnósticas e de registro, acesso à assistência médica e tratamento e questões sociais do público em estudo.
Todos os autores declararam não haver qualquer potencial conflito de interesses referente a este artigo.

\section{REFERÊNCIAS}

AMORIM, Vivian Mae Schmidt Lima et al. Fatores associados à realização dos exames de rastreamento para o câncer de próstata: um estudo de base populacional: um estudo de base populacional. Cadernos de Saúde Pública, v. 27, n. 2, p. 347356, 2011.

ANTUNES, Yuri Philippe Pimentel Vieira et al. Características clínicas e de sobrevida global em pacientes oncológicos idosos num centro oncológico terciário. Einstein, v.13, n. 4, p. 487491, 2015.

BRAGA, Sonia Faria Mendes et al. Patient survival and risk of death after prostate cancer treatment in the Brazilian Unified Health System. Revista de Saúde Pública, v. 51, p. 1-10, 2017.

BRASIL. MINISTÉRIO DA SAÚDE. DATASUS. Informações de Saúde: Mortalidade 2010-2015. 2016. Rio de Janeiro; [Acesso em: 20 março 2018]. Disponível em: http://sim.saude.gov.br.

BRASIL. Ministério da Saúde. Secretaria de Atenção à Saúde. Política Nacional de Atenção Integral à Saúde do Homem: princípios e diretrizes. Brasília: Ministério da Saúde, 2009. p. 7-9.

BRAY, Freddie et al. Global cancer statistics 2018: globocan estimates of incidence and mortality worldwide for 36 cancers in 185 countries. Ca: A Cancer Journal for Clinicians, v. 68, n. 6, p. 394424, 13 mai. 2021.

CZORNY, Rildo César Nunes et al. Fatores de risco para o câncer de próstata: população de uma unidade básica de saúde. Cogitare Enfermagem, v. 22, n. 4, 2017.

DAMIÃO, Ronaldo et al. Câncer de próstata. Revista Hospital Universitário Pedro Ernesto, v. 14, n. 1, p. 80-86, 2015.

GUERRA, Maximiliano Ribeiro et al. Risco de câncer no Brasil: tendências e estudos epidemiológicos mais recentes. Revista Brasileira de Cancerologia, Rio de Janeiro, v.51, n.3, p. 227234, 2008. 
INSTITUTO BRASILEIRO DE GEOGRAFIA E ESTATÍSTICA - IBGE. Cidades e estados do Brasil [Acesso em: 17 ago. 2020]. 2019. Disponível em: https://cidades.ibge.gov.br/

INSTITUTO NACIONAL DE CÂNCER (INCA). CÂNCER: Magnitude do problema. 2018. Rio de Janeiro; [Acesso em: 16 dez. 2019]. Disponível em: https://www.femama.org.br/2018/br/noticia/oproblema-da-magnitude-do-cancer-no-mundo.

INSTITUTO NACIONAL DE CÂNCER (INCA). Estimativa: incidência de câncer no Brasil. 2015. Rio de Janeiro; [Acesso em: 20 dez. 2019]. Disponível em: https://www.inca.gov.br/tipos-decancer/cancer-de-prostata.

INSTITUTO NACIONAL DE CÂNCER (INCA). Estimativa: incidência de câncer no Brasil. 2020. Rio de Janeiro; [Acesso em: 20 dez. 2019]. Disponível em: https://www.inca.gov.br/estimativa.

LIMA, Alisson Padilha de et al. Prevalence and factors associated with the performance of prostate cancer screening in the elderly: a population-based study: a population-based study. Revista Brasileira de Geriatria e Gerontologia, v. 21, n. 1, p. 53-59, 2018.

MEDEIROS, Adriane Pinto de; MENEZES, Maria de Fátima Batalha de; NAPOLEAO, Ana Maria Alves. Fatores de risco e medidas de prevenção do câncer de próstata: subsídios para a enfermagem. Rev. bras. enferm., v. 64, n. 2, p. 385-388, 2011.

REGISTROS HOSPITALARES DE CÂNCER (RHC). 2015. Rio de Janeiro; [Acesso em: 18 fev. 2018]. Disponível em: https://irhc.inca.gov.br/.

SANTOS, S. M.; SOUZA, W. V. Introdução à Estatística Espacial para a Saúde Pública. Brasília: Ministério da Saúde, 2007.

SANTOS JÚNIOR, C. J.; OLIVEIRA, E. C. T. Morbimortalidade por câncer de próstata em homens adultos em um estado do Nordeste do Brasil: caracterização epidemiológica e análise de tendência temporal. Revista Brasileira de Ciências da Saúde, v. 24, n. 4, p. 1-12, 5 dez. 2020.

SECRETARIA DE ESTADO DA SAÚDE SEPLAG. Regiões de Saúde do Estado de Alagoas [Acesso em: 17 ago. 2020]. 2019. Disponível em: <http://dados.al.gov.br/>.

SOCIEDADE BRASILEIRA DE UROLOGIA (SBU). Nota Oficial: Rastreamento do Câncer de Próstata. 2018. [Acesso em: 25 dez. 2018].
Disponível em: https://portaldaurologia.org.br/medicos/destaquesbu/nota-oficial-2018-rastreamento-do-cancer-deprostata/.

SOUZA, Karolayne Silva et al. Tendência de mortalidade por câncer de próstata na região Nordeste do Brasil, 1996-2014. Bases Conceituais da Saúde, v. 7, p. 255-262, 2019.

SOUZA, N. P. de; SILVA, E. M. G. C.; TEIXEIRA, M. D.; LEITE, L. R. Aplicação do Estimador de Densidade Kernel em Unidades de Conservação na Bacia do Rio São Francisco para análise de focos de desmatamento e focos de calor. 4958-4965. 2013. In: Anais XVI Simpósio Brasileiro de Sensoriamento Remoto - SBSR, Foz do Iguaçu, PR, Brasil, 13 a 18 de abril de 2013, INPE.

TSODIKOV, Alex et al. Reconciling the Effects of Screening on Prostate Cancer Mortality in the ERSPC and PLCO Trials. Annals Of Internal Medicine, v. 167, n. 7, p. 449-455, 2017.

VIANA, Marina et al. Perfil epidemiológico do homem com câncer de próstata atendido em um hospital universitário. Cogitare Enfermagem, v. 19, n. 2, p. 333-340, 30 jun. 2014.

WORLD HEALTH ORGANIZATION (WHO). World Cancer Report 2020. Lyon: World Health Organization; 2020. 